Domingo-Coscolla, M., Bosco, A., Carrasco Segovia, Sy Sánchez Valero, J. A. (2020) Fomentando la competencia digital docente en la universidad: Percepción de estudiantes y docentes. Revista de Investigación Educativa, 38(1), 167-782. DOI: http://dx.doi.org/10.6018/rie.340551

\title{
Fomentando la competencia digital docente en la universidad: Percepción de estudiantes y docentes
}

\author{
Fostering teacher's digital competence at university: The \\ perception of students and teachers
}

\author{
María Domingo-Coscollola*,Alejandra Bosco**, Sara Carrasco Segovia*** y Joan-Anton \\ Sánchez Valero**** \\ *Universitat Internacional de Catalunya \\ **Universitat Autònoma de Barcelona \\ ****Universitat de Barcelona
}

\begin{abstract}
Resumen
Este artículo da cuenta del proyecto "Propuesta interuniversitaria de formación inicial de maestros en tecnologías digitales." Para elaborarla, nos planteamos conocer la percepción de estudiantes y de docentes sobre qué necesidades abordar en relación con las tecnologías digitales y la competencia digital metodológica, y qué acciones institucionales son preferentes para desarrollar la competencia digital docente (CDD). En este estudio, utilizamos un diseño de investigación de métodos mixtos. Las técnicas e instrumentos usados han sido el análisis de la documentación disponible, grupos de discusión y cuestionarios. La propuesta va dirigida a las nueve universidades catalanas que imparten la formación de futuros docentes en los grados de Maestro de Educación Infantil y Primaria. Las principales conclusiones apuntan hacia la necesidad de vincular la universidad con la sociedad, así como de favorecer un desarrollo profesional del profesorado universitario y la alfabetización digital de sus estudiantes fomentando el aprendizaje colaborativo y la autoría. También, se destaca la importancia de priorizar la comunicación y la colaboración durante el proceso de enseñanza y aprendizaje usando recursos digitales útiles que lo faciliten. A su vez, se resalta la ética y la ciudadanía digital como una dimensión emergente
\end{abstract}

Correspondencia: María Domingo-Coscollola. mdomingoc@uic,es Terré 11-19, 08017, Barcelona. 
a considerar en la práctica educativa. Finalmente, se apuntan tres acciones institucionales sobre CDD para contemplar en los planes de estudio universitarios.

Palabras clave: competencia digital docente; competencia digital metodológica; educación superior; formación inicial de maestros; formación de futuros docentes; tecnologías digitales.

\section{Abstract}

This article deals with the project "An inter-university proposal for the initial training of teachers in digital technologies." In order to prepare it, we examine the views of students and teachers on the issues with digital technologies and methodological digital competence along with the appropriate institutional measures to develop teachers' digital competence (TDC). In this study, we used a mixed-method research design. The main techniques and instruments used in the present study were the analysis of available information, focus groups and surveys. The proposal is targeted at the nine Catalan Universities that provide training for future teachers in Pre-school and Primary Education Degrees. The main findings point to the need to link the university to society, as well as to promote a professional development of university teachers and the digital literacy of their students by fostering collaborative learning and authorship. Likewise, it highlights the importance of prioritizing communication and cooperation during the teaching and learning process using useful digital resources that facilitate it. At the same time, the present study highlights ethics and digital citizenship as an emerging dimension that needs to be considered in educational practice. Finally, three institutional actions about TDC are pointed out to consider in university curricula.

Keywords: teachers' digital competence; methodological digital competence; higher education; initial teacher training; future teachers' training; digital technologies.

\section{Introducción}

En las últimas décadas, las tecnologías de la información y la comunicación (TIC) están cambiando las prácticas de alfabetización en nuestra sociedad (Cela-Ranilla, EsteveGonzález, Esteve-Mon, González-Martínez, y Gisbert-Cervera, 2017; Guzmán-Simón, García-Jiménez, y López-Cobo, 2017; Suárez-Rodríguez, Almerich, Gargallo-López, y Aliaga, 2013). Esta realidad debería estar contemplada en la formación de docentes y de futuros docentes para incidir en su actividad profesional (Batane y Ngwako, 2017; Instefjord y Munthe, 2017; Miño-Puigcercós, Domingo-Coscollola, y Sancho-Gil, 2019; Sancho-Gil, Ornellas, y Arrazola, 2018; Spiteri y Chang-Rundgren, 2017; Tondeur, Scherer, Siddiq, y Baran, 2017). Sobre esta formación, la UNESCO (2011, p. 3) señala que no es suficiente que el profesorado tenga competencias TIC y las enseñe al alumnado, sino que también debería dominar los instrumentos digitales para que sus alumnos puedan convertirse en ciudadanos autónomos, integrados en la sociedad y con capacidad de seguir aprendiendo. Este planteamiento requiere usar metodologías activas para conseguirlo.

Fruto de esta actual realidad, se está configurando una nueva capacidad: la competencia digital docente (CDD). Según Castañeda, Esteve, y Adell (2018, p. 14) es "una competencia docente para el mundo digital entendida como holística, situada, orientada hacia roles de desempeño, función y relación, sistémica, entrenable y en 
constante desarrollo". Esta CDD viene conformada por las habilidades, actitudes y conocimientos requeridos por los educadores para apoyar el aprendizaje del alumno como participante activo en un mundo digital (Hall, Atkins, y Fraser, 2014). También, se define como el conjunto de capacidades y habilidades que nos lleven a incorporar y usar adecuadamente las TIC como recurso metodológico convirtiéndose en Tecnologías para el Aprendizaje y el Conocimiento (TAC) con una clara implicación didáctica (Tourón, Martín, Navarro, Pradas, e Íñigo, 2018).

Actualmente, el dominio de la CDD incide en la profesionalidad del profesorado. Tal como indican Gudmundsdottir y Hatlevik (2018), la competencia digital profesional tiene una importancia creciente en una realidad donde los recursos y medios digitales forman parte de la práctica educativa diaria. Así, se espera que las facultades del grado de Maestro preparen al alumnado proporcionando la CDD necesaria para su futura práctica docente (Gudmundsdottir y Hatlevik, 2018). Para conseguirlo, "la necesidad de una mejor preparación inicial profesional debería ser una prioridad" (Sancho-Gil, Sánchez-Valero, y Domingo-Coscollola, 2017, p. 1).

En distintos países, se han realizado y se están actualizando iniciativas para asegurar la CDD y desarrollar la competencia digital del alumnado. Por ejemplo, a nivel español, el Marco Común de Competencia Digital Docente del Instituto Nacional de Tecnologías Educativas y Formación de Profesorado (INTEF, 2017) la estandariza en 5 áreas (información y alfabetización informacional, comunicación y colaboración, creación de contenidos digitales, seguridad, y resolución de problemas). También, existen modelos internacionales como el propuesto por la UNESCO (2011) o por la International Society for Technology in Education (ISTE, 2018) actualizado recientemente. A nivel europeo, la Comisión Europea ha publicado el Marco Europeo para Organizaciones Educativas Competentes Digitalmente: DigCompOrg (Kampylis, Punie, y Devine, 2015) y el Marco de la Competencia Digital del profesorado: DigCompEdu (Redecker y Punie, 2017).

En el caso de las facultades de educación españolas, existe la preocupación de promover la competencia digital del profesorado y del alumnado (Gutiérrez, Palacios, y Torrego, 2010) y también de conseguir que el alumnado pueda "desarrollarla posteriormente en sus futuros estudiantes" (Ruiz, Rubia, Anguita, y Fernández, 2010, p. 173). A su vez, se va destacando que el alumnado del grado de Maestro no recibe una formación adecuada en relación a las tecnologías digitales (Herrada y Herrada, 2011; Losada, Valverde, y Correa, 2012; Paredes, Guitert, y Rubia, 2015; Sancho-Gil, Bosco, Alonso y Sánchez, 2015). Actualmente, esta formación inadecuada sigue existiendo. Por ejemplo, "en general, las prácticas académicas de la universidad española no incorporan procesos de alfabetización en información y TIC como parte de la alfabetización académica de los estudiantes" (Guzmán-Simón et al., 2017, p. 202).

En este contexto español (fruto de la transferencia de competencias en educación), las comunidades autónomas asumen el reto de concretar la capacitación docente de la formación inicial y del profesorado en activo. En Cataluña, la CDD se contempla como la capacidad que el profesorado tiene para movilizar y transferir todos sus conocimientos, estrategias, habilidades y actitudes sobre el uso de las TAC durante su praxis profesional (DEGC, 2016, 2018). Para conseguir esta movilización y transferencia, se demanda: 
(a) facilitar el aprendizaje de los alumnos y la adquisición de la competencia digital de este colectivo; (b) llevar a cabo procesos de mejora e innovación en la enseñanza de acuerdo con las necesidades de la era digital; y (c) contribuir a su desarrollo profesional de acuerdo con los procesos de cambio que tienen lugar en la sociedad y en los centros educativos (DEGC, 2016, p. 2; 2018, p. 11).

Así, la CDD hace referencia a la competencia digital instrumental (CDI) y también a la competencia digital metodológica (CDM). Este planteamiento está en línea con Krumsvik (2014) referente a que los docentes requieren alfabetización digital básica y capacidad de incorporar la tecnología en su praxis de enseñanza para favorecer el aprendizaje.

En las universidades catalanas con grado de Maestro, la competencia digital tiene tratamientos diferentes en sus planes de estudio (a nivel de asignaturas, contenidos y planteamientos) y el profesorado ofrece un tratamiento desigual en la práctica educativa (Sancho-Gil et al., 2015). Fruto de esta realidad planteada, se presenta el proyecto "Propuesta interuniversitaria de Formación Inicial de Maestros en Tecnologías Digitales (FIMTD)" en la convocatoria ARMIF de 2015 de la Generalitat de Cataluña. Desde este proyecto, nos planteamos recoger percepciones de estudiantes y docentes sobre: (1) qué necesidades abordar en relación a las tecnologías digitales, (2) qué es prioritario de la CDM y (3) qué acciones institucionales son preferentes para desarrollar la CDD en los grados de Maestro.

\section{Método}

En el proyecto FIMTD, participan investigadores de once universidades catalanas: Universitat Autònoma de Barcelona (UAB), Universitat Abat Oliba (UAO), Universitat de Barcelona (UB), Universitat de Girona (UdG), Universitat de LLeida (UdL), Universitat Internacional de Catalunya (UIC), Universitat Oberta de Catalunya (UOC), Universitat Pompeu Fabra (UPF), Universitat Ramon LLull (URL), Universitat Rovira i Virgili (URV) y Universitat de Vic (UVic). La finalidad del proyecto es elaborar una propuesta de formación inicial de maestros sobre la CDD para las nueve universidades catalanas que imparten el grado de Maestro (UAB, UAO, UB, UdG, UdL, UIC, URL, URV y UVic). En este estudio, utilizamos un diseño de investigación de métodos mixtos (Creswell, 2009). Las técnicas e instrumentos usados fueron el análisis de la documentación disponible, grupos de discusión y cuestionarios. Con el fin de orientar cómo ir transformando las prácticas de enseñanza y aprendizaje en la universidad, hemos implicado a diferentes colectivos y hemos contemplado sus aportaciones en esta propuesta de cambio.

Inicialmente, se llevaron a cabo 3 grupos de discusión desarrollados simultáneamente en la UB dirigidos por investigadores del proyecto (en marzo de 2017) siguiendo el método de grupos de discusión de Krueger y Casey (2014). La discusión se focalizó en torno a dos preguntas sobre la formación de futuros docentes: ¿Qué necesidades son prioritarias en relación a las tecnologías digitales? y ¿Qué acciones institucionales son prioritarias para el desarrollo de la CDD? En cada uno de estos grupos, participaba un colectivo diferente. Un grupo estaba formado por estudiantes de $4^{\circ}$ curso de cada una de las 9 universidades catalanas con grado de Maestro. Otro, por docentes de educación primaria en activo de las 4 provincias catalanas con perfil TIC y/o TAC en su centro. Y el último, por docentes de 5 redes de referencia en Cataluña sobre formación de profesorado. 
Concretamente, en el grupo de discusión de estudiantes, participaron 11 personas: dos estudiantes de la UAB y de la UB (universidades con mayor número de estudiantes) y uno de cada una de las siete universidades restantes. En el grupo de discusión de docentes con perfil TIC y/o TAC, participaron 10 personas: 4 de Barcelona (la provincia más poblada), 2 de Gerona, 2 de Tarragona y 2 de Lérida. Y en el grupo de discusión de docentes de redes de docentes, también participaron 10 personas: 3 docentes de la Xarxa de Competències Bàsiques, 3 de la Federació de Moviments de Renovació Pedagògica, 2 de la Associació Espiral, 1 de la Associació de Mestres Rosa Sensat y 1 de IEARN-Pangea. Las evidencias se recogieron a través de la grabación en vídeo y de la transcripción de las conversaciones.

Posteriormente, se elaboró un cuestionario de preguntas cerradas con ítems (ver tabla 1) de las 5 dimensiones de la CDM y sus 26 descriptores definidos por el DEGC (2016, pp. 3-4). Los cuestionarios se realizaron digitalmente mediante la herramienta de Formularios de Google. Cada ítem se podía valorar según el grado de importancia para la formación, en los grados de Maestro, mediante una escala de Likert de cinco puntos. Respondieron un total de 1.051 docentes y de 473 estudiantes entre los meses de mayo y septiembre de 2017. El análisis de datos se hizo con RStudio. Se ordenaron las dimensiones y los descriptores de mayor a menor relevancia según ambos colectivos. El número de respuestas obtenidas representa un $95 \%$ de confiabilidad en ambos casos, con margen de error de 4 para los docentes y de 3 para los estudiantes. Estas respuestas permitieron conocer qué dimensiones y descriptores de la CDM son prioritarios (para desarrollar en la práctica educativa) según la valoración de docentes en activo en las escuelas y de estudiantes del último curso de las facultades catalanas de formación de futuros maestros.

Tabla 1

Dimensiones y descriptores de la CDM definidos por el DEGC (2016, pp. 3-4)

\begin{tabular}{|c|c|}
\hline Dimensión & Descriptores \\
\hline \multirow{7}{*}{$\begin{array}{l}\text { Diseño, } \\
\text { planificación e } \\
\text { implementación } \\
\text { didáctica (1) }\end{array}$} & $\begin{array}{l}\text { Uso de las tecnologías digitales como recursos y estrategias en procesos de ense- } \\
\text { ñanza y aprendizaje (1.1). }\end{array}$ \\
\hline & $\begin{array}{l}\text { Selección de recursos digitales para el diseño de actividades y la planificación didác- } \\
\text { tica (1.2) }\end{array}$ \\
\hline & $\begin{array}{l}\text { Incorporación de tecnologías digitales en coherencia con el proyecto educativo y las } \\
\text { infraestructuras del centro (1.3) }\end{array}$ \\
\hline & $\begin{array}{l}\text { Incorporación de la competencia digital de los alumnos a las programaciones didác- } \\
\text { ticas (1.4) }\end{array}$ \\
\hline & Uso de las tecnologías digitales para atender la diversidad de los alumnos (1.5) \\
\hline & Uso de las tecnologías digitales en el seguimiento y la evaluación de los alumnos (1.6) \\
\hline & Aplicación de metodologías innovadoras con el uso de tecnologías digitales (1.7) \\
\hline
\end{tabular}




\begin{tabular}{|c|c|}
\hline Dimensión & Descriptores \\
\hline \multirow{4}{*}{$\begin{array}{l}\text { Organización y } \\
\text { gestión de espacios } \\
\text { y recursos digitales } \\
\text { (2) }\end{array}$} & $\begin{array}{l}\text { Conocimiento y aplicación de las normas de uso de los recursos, infraestructuras y } \\
\text { espacios digitales (2.1) }\end{array}$ \\
\hline & Conocimiento y uso del software de aplicación general del centro (2.2) \\
\hline & $\begin{array}{l}\text { Organización de las tecnologías digitales teniendo en cuenta los diferentes ambien- } \\
\text { tes de aprendizaje (2.3) }\end{array}$ \\
\hline & Implicación en proyectos de centro relacionados con las tecnologías digitales (2.4) \\
\hline \multirow{3}{*}{$\begin{array}{l}\text { Comunicación y } \\
\text { colaboración } \\
\text { (3) }\end{array}$} & Comunicación utilizando tecnologías digitales (3.1) \\
\hline & Participación activa en redes educativas en entornos digitales (3.2) \\
\hline & Fomento de la construcción colaborativa de conocimiento con recursos digitales (3.3) \\
\hline \multirow{5}{*}{$\begin{array}{l}\text { Ética y } \\
\text { civismo digital } \\
\text { (4) }\end{array}$} & $\begin{array}{l}\text { Protección de los derechos fundamentales a la intimidad personal y a la propia ima- } \\
\text { gen en el uso de las tecnologías digitales (4.1) }\end{array}$ \\
\hline & Uso responsable, seguro y saludable de las tecnologías digitales (4.2) \\
\hline & Promoción del acceso a los recursos respetando la propiedad intelectual (4.3) \\
\hline & Fomento de la inclusión digital (4.4) \\
\hline & Fomento de la construcción de una adecuada identidad digital (4.5) \\
\hline \multirow{7}{*}{$\begin{array}{l}\text { Desarrollo } \\
\text { profesional } \\
(5)\end{array}$} & Configuración de la propia identidad digital profesional (5.1) \\
\hline & $\begin{array}{l}\text { Práctica reflexiva sobre la actividad profesional relacionada con las tecnologías digi- } \\
\text { tales (5.2) }\end{array}$ \\
\hline & Incorporación de innovaciones docentes basadas en las tecnologías digitales (5.3) \\
\hline & $\begin{array}{l}\text { Participación en investigaciones educativas relacionadas con las tecnologías digitales } \\
\text { (5.4) }\end{array}$ \\
\hline & Creación y divulgación de contenidos y recursos educativos en formato digital (5.5) \\
\hline & $\begin{array}{l}\text { Participación en comunidades virtuales de aprendizaje para la actualización docente } \\
\text { (5.6) }\end{array}$ \\
\hline & $\begin{array}{l}\text { Participación en actividades de formación permanente en el ámbito de la competen- } \\
\text { cia digital (5.7) }\end{array}$ \\
\hline
\end{tabular}

\section{Resultados}

En este apartado, recogemos los principales resultados organizados en torno a:

- Necesidades prioritarias sobre las tecnologías digitales en la práctica educativa.

- Dimensiones y descriptores de la CDM prioritarios durante la práctica educativa.

- Acciones institucionales preferentes para desarrollar la CDD en los grados de Maestro. 


\section{Necesidades prioritarias sobre las tecnologías digitales en la práctica educativa}

Se presentan los resultados sobre qué se debería priorizar en relación a las tecnologías digitales durante la práctica educativa de la formación inicial de maestros. Estos resultados surgen de las valoraciones de los tres grupos de discusión realizados.

En el grupo de discusión de docentes de redes de docentes, las necesidades prioritarias para la práctica educativa fueron:

- Conectar la universidad con la escuela y la sociedad. "Estar atento a las nuevas posibilidades y cómo éstas puedan mejorar las prácticas" (Participante 4D-R). “La gente de prácticas (...) no conoce cómo planificar ni cómo aplicar las tecnologías (...) ni por qué las están utilizando ni por qué están haciendo lo que están haciendo. Tampoco sabe el cambio metodológico que se está produciendo en las escuelas" (Participante 6D-R).

- Potenciar la comunicación, la autoría y el aprender con tecnologías digitales: "Deben saber hacer comunidad virtual a través de redes sociales participando. Es un proceso en el cual puedes actualizarte a nivel de herramientas y puedes crear comunidad y participar, y donde más y más funciona el autoaprendizaje" (Participante 2D-R). "Estamos intentando utilizar algunas metodologías dentro del aula que le den más protagonismo al alumno, que el alumno sea realmente quien gestione su propio aprendizaje con un rol activo (...) capaz de aplicar y transferir todas sus habilidades y conocimientos que tiene como propios" (Participante 7D-R).

- Tener una visión crítica ante las tecnologías digitales: “Conocer las tecnologías digitales comprende criticar y ser crítica (...) y, por tanto, saber si eso funciona mejor o peor" (Participante 3D-R). "El profesor o maestro ha de ser capaz de distinguir cuando una herramienta nueva aporta alguna cosa significativa a lo que ya está hecho" (Participante 8D-R). "El punto crítico frente a las tecnologías ha de ser muy importante. Porque si no, sólo seguimos una moda" (Participante 8D-R).

- Incidir en el conocimiento metodológico de las tecnologías digitales y no sólo en el conocimiento técnico: “Es mucho más importante que encontrar la aplicación, saber cómo aplicarla para aprender o enseñar (...) las herramientas se aplican mal" (Participante 1D-R). "Es muy importante el tema sobre replantear las metodologías teniendo en cuenta que las tecnologías nos ofrecen nuevas posibilidades de cómo diseñar, planificar, evaluar y comunicarse. Las competencias digitales van más ligadas a temas pedagógicos que a instrumentales" (Participante 5D-R).

- En el grupo de docentes con perfil TIC y/o TAC en su centro, las necesidades prioritarias para la práctica educativa fueron:

- Usar las tecnologías digitales como recursos y estrategias en procesos de enseñanza y aprendizaje: "Yo creo que la clave está en integrar las tecnologías en las actividades del día a día" (Participante 5D-T). "Las nuevas tecnologías permiten mucho estos tipos de trabajo en equipo: espacios de trabajos en redes sociales, redes sociales educativas, entornos virtuales de aprendizaje, cuestiones que deberían ser útiles en el día a día" (Participante 2D-T). "Necesitamos 
también una sensibilización de qué tipo de chicos, chicas y jóvenes hemos de formar y en qué tipo de sociedad ellos y ellas deberán moverse: una sociedad digital" (Participante 8D-T).

- Fomentar la creación y la autoría con recursos digitales en grupo: "Han de tener muy claro que han de estar abiertos a compartir, a trabajar en grupo para crear y realizar trabajos. Para eso, tenemos unas herramientas básicas que se han de utilizar en la facultad" (Participante 3D-T). "Tenemos que tener la capacidad de salir a buscar lo que no nos han enseñado. Tener ganas, acercarnos a las redes, compartir, ir a jornadas participando activamente" (Participante 6D-T).

- Aprender de forma colaborativa: "No han de tener miedo, sino ganas de aprender con los otros" (Participante 9D-T). "Crear redes con profesores activos, compartir, hacer investigación propia... no es más importante la herramienta o la aplicación, sino el modo de trabajar y la competencia que ésta da" (Participante 1D-T).

- Formar la creatividad de los estudiantes: “La creatividad no necesariamente quiere decir crear un producto o una aplicación, sino ser creativo con una aplicación que quizás ya existe" (Participante 4D-T). "Me gusta mucho la idea de formar a los alumnos con capacidad de crear creativamente, porque si miramos lo que se pediría en una mención en tecnologías estaría: vídeo digital, diseño y creación de material, imagen digital en contexto, sonido digital, mass media, desarrollar Apps, etc." (Participante 1D-T).

Y en el grupo de estudiantes, las necesidades prioritarias para la práctica educativa fueron:

- Desfase entre la universidad y la escuela. "En mi escuela, los niños le enseñan a la profesora cómo usar una pizarra digital. Ahora que tenemos recursos tecnológicos en las escuelas, no pueden quedar sin utilizarse por no saber cómo" (Participante 3E). "Se nota mucho cuando un profesor está conectado con la escuela, porque te enseña muy bien la teoría y también cómo llevarla a la práctica" (Participante 7E).

- Incorporar los recursos digitales en la planificación didáctica: "Hay una parte que es más técnica y otra que tiene que ver con la didáctica en general, y una vez lo tienes claro, puedes unirlas" (Participante 8E). "Yo creo que se debería dedicar más tiempo al uso de las tecnologías en las asignaturas de didácticas que hacemos de lenguas, ciencias sociales y naturales" (Participante 2E). "Puedes llevar el uso de las TIC a muchas asignaturas" (Participante 3E).

- Trabajar el civismo digital: "La ética es una cuestión básica que hay que considerar para todo" (Participante 5E). "Hay que tener mucho cuidado con la utilización de la imagen. Es necesario enseñarlo y cuidar la identidad de los niños con los que se trabaja" (Participante 6E).

\section{Dimensiones y descriptores de la CDM prioritarios durante la práctica educativa}

Se presentan los resultados obtenidos desde los cuestionarios realizados sobre qué dimensiones y descriptores de la CDM son prioritarios (ver tabla 2). Según los docen- 
tes, la dimensión más priorizada fue comunicación y colaboración, seguida de ética y civismo digital. En el caso de los estudiantes, los resultados fueron parecidos excepto en la dimensión de desarrollo profesional que obtuvo una puntuación superior a la de ética y civismo digital.

Tabla 2

Resultados sobre la priorización de las dimensiones según docentes y estudiantes

\begin{tabular}{lcc}
\hline \multicolumn{1}{c}{ Dimensiones (con $\mathbf{n}^{\mathbf{0}}$ identificador) } & \multicolumn{2}{c}{ Priorización según } \\
Docentes & Estudiantes \\
\hline Comunicación y colaboración (3) & 4.02 & 4.06 \\
\hline Ética y civismo digital (4) & 4.00 & 3.92 \\
\hline Desarrollo profesional (5) & 3.98 & 4.00 \\
\hline Diseño, planificación e implementación didáctica (1) & 3.91 & 3.83 \\
\hline Organización y gestión de espacios y recursos digitales (2) & 3.87 & 3.72 \\
\hline
\end{tabular}

Los docentes y los estudiantes coincidieron en señalar nueve descriptores prioritarios y normalmente con el mismo orden (ver tabla 3). Destacamos que, de estos nueve, cinco pertenecen a la dimensión de ética y civismo digital y además tres de ellos ocupan las tres primeras posiciones como prioritarios: el uso responsable, seguro y saludable de las tecnologías digitales (4.2), la protección de los derechos a la intimidad personal y a la propia imagen en el uso de las tecnologías digitales (4.1), y la promoción del acceso a los recursos respetando la propiedad intelectual (4.3). Posteriormente, se prioriza el fomento de la inclusión digital (4.4) y de la construcción de una adecuada identidad digital (4.5).

Tabla 3

Resultados sobre la priorización de los descriptores según docentes y estudiantes

\begin{tabular}{lcc}
\hline \multicolumn{1}{c}{ Descriptores (con no $\mathbf{~ i d e n t i f i c a d o r ) ~}^{-}$} & \multicolumn{2}{c}{ Priorización según } \\
Docentes & Estudiantes \\
\hline $\begin{array}{l}\text { Uso responsable, seguro y saludable de las tecnologías digi- } \\
\text { tales (4.2) }\end{array}$ & 4.38 & 4.31 \\
\hline $\begin{array}{l}\text { Protección de los derechos a la intimidad personal y a la } \\
\text { propia imagen en el uso de las tecnologías digitales (4.1) }\end{array}$ & 4.32 & 4.18 \\
\hline $\begin{array}{l}\text { Promoción del acceso a los recursos respetando la propie- } \\
\text { dad intelectual (4.3) }\end{array}$ & 4.08 & 3.99 \\
\hline $\begin{array}{l}\text { Uso de las tecnologías digitales como recursos y estrategias } \\
\text { en procesos de enseñanza y aprendizaje (1.1) }\end{array}$ & 4.06 & 3.83 \\
\hline
\end{tabular}




\begin{tabular}{lcc}
\hline \multicolumn{1}{c}{ Descriptores (con $\mathbf{n}^{\mathbf{0}}$ identificador) } & \multicolumn{2}{c}{ Priorización según } \\
& Docentes & Estudiantes \\
\hline Fomento de la inclusión digital (4.4) & 4.01 & 3.82 \\
\hline Comunicación utilizando tecnologías digitales (3.1) & 4.00 & 3.79 \\
\hline $\begin{array}{l}\text { Incorporación de tecnologías digitales en coherencia con el } \\
\text { proyecto educativo y las infraestructuras del centro (1.3) }\end{array}$ & 3.99 & 3.78 \\
\hline $\begin{array}{l}\text { Fomento de la construcción de una adecuada identidad } \\
\text { digital (4.5) }\end{array}$ & 3.98 & 3.96 \\
\hline $\begin{array}{l}\text { Selección de recursos digitales para el diseño de actividades } \\
\text { y la planificación didáctica (1.2) }\end{array}$ & 3.98 & 3.77 \\
\hline
\end{tabular}

\section{Acciones preferentes para desarrollar la CDD en los grados de Maestro}

Se presentan los resultados sobre qué acciones institucionales se deberían priorizar para el desarrollo de la CDD en los grados de Maestro. Estos resultados surgen de las valoraciones de los tres grupos de discusión realizados.

En el grupo de discusión de docentes de redes de docentes, las acciones priorizadas fueron:

- Incluir en el plan de estudios una asignatura obligatoria de CDD: "Lo que nosotros proponemos es una asignatura para presentar nuevos escenarios didácticos donde la tecnología pueda tener un papel importante que replantee las maneras de aprender, evaluar y comunicarse" (Participante 3D-R). "Hacer una asignatura específica para iniciar a fondo todo este trabajo metodológico de las competencias digitales y que, después, las puedan ir consolidando a lo largo del grado" (Participante 1D-R).

- Trabajar la CDD y validarla por igual desde el trabajo de final de grado o desde una asignatura: "En el trabajo de final de grado o en una asignatura, se debería evaluar la competencia digital” (Participante 6D-R).

- Implementar la competencia digital en las asignaturas de didáctica: "Yo creo que la dimensión digital ha de ser una dimensión transversal" (Participante 8D-R). "Es importante trabajar la competencia digital desde las diferentes didácticas" (Participante 5D-R).

En el grupo de discusión de docentes con perfil TIC y/o TAC, las acciones priorizadas fueron:

- Incorporar en el plan de estudios una asignatura obligatoria de CDD: "Yo creo que tan importante es que estén formados en las tecnologías como en las metodologías desde una asignatura específica" (Participante 4D-T). "Debería haber (...) una en primero, pero tenerla como un pilar y una base sólida. Luego que los maestros de las demás asignaturas, por ejemplo de didáctica, que apliquen lo de la asignatura de base" (Participante 1D-T). 
- Abordar aspectos tecnológicos y metodológicos en una asignatura obligatoria: “Un alumno que salga del grado ha de tener conocimientos metodológicos y estar abierto a todos estos cambios tecnológicos. Esto requiere estar planificado desde una asignatura" (Participante 2D-T). "Yo creo que se ha de asociar el tema pedagógico y el metodológico" (Participante 5D-T). “La propuesta de formación sí que tendría que centrarse en conocer algunas herramientas, pero también en la implicación que éstas tienen en la programación metodológica" (Participante 8D-T).

En el grupo de discusión de estudiantes, las acciones priorizadas fueron:

- Incluir las tecnologías digitales en las asignaturas de didáctica como un tratamiento transversal durante la titulación: "Yo creo que se debería dedicar más tiempo al uso de las tecnologías en las asignaturas de didáctica que hacemos de lenguas, de ciencias sociales y naturales" (Participante 6E).

- Incluir una asignatura específica de CDD: "Está bien tener una asignatura específica a nivel de base" (Participante 8E). "Crear una asignatura específica y que ésta debería tener continuidad" (Participante 3E).

- Unificar los planes de estudio entre las universidades y exigir la CDD al terminar el grado validándola: “No te damos el grado si no adquieres $X$ competencias digitales validadas desde una mención o una asignatura" (Participante 2E). "Se debería validar, al igual que te exigen un C2 de catalán o un B2 de inglés" (Participante 5E).

\section{Discusión y conclusiones}

El análisis realizado de los resultados desvela indicaciones sobre la CDD durante la formación de los grados de Maestro. Destacamos las percepciones significativas obtenidas relacionándolas con distintos estudios y propuestas internacionales:

- La formación universitaria se debería sincronizar con la escuela y la sociedad. En general, la escuela está poniendo énfasis en hacer cambios mientras que la universidad sigue repitiendo prácticas tradicionales. Batane y Ngwako (2017, p. 58) señalan la conveniencia de establecer un fuerte vínculo entre la educación superior y lo que ocurre en la escuela. Por ejemplo, se destaca un aprendizaje transdisciplinar, colaborativo y compartido en educación primaria (DomingoCoscollola, Onsès-Segarra, y Sancho-Gil, 2018). A su vez, se acentúa "una gran brecha entre la competencia digital desarrollada en contextos de aprendizaje informal y su escasez en las prácticas de alfabetización universitaria" (GuzmánSimón et al., 2017, p. 202). Por lo tanto, en la práctica educativa, generalmente "la formación docente no está en sintonía con lo que está sucediendo en el mundo real" (Sancho-Gil et al., 2017, p. 2).

- El dominio de la CDD del profesorado universitario es deficiente. Generalmente, menor en la CDM que en la CDI. Diferentes estudios revelan esta problemática. Por ejemplo, "el profesorado posee un nivel de competencias en TIC medio, mayor en las tecnológicas que en las pedagógicas" (Suárez-Rodríguez et al., 2013, 
p. 51). Esta carencia tiene repercusiones: "las alfabetizaciones deficientes en TIC e información pueden convertirse en el origen de dificultades para el desarrollo profesional como docentes" (Guzmán-Simón et al., 2017, p. 202). Por lo tanto, se debería situar el dominio "de la CDD dentro del conjunto de requerimientos (...) del ejercicio de la profesión de docente" (Cela-Ranilla et al., 2017, p. 407).

- La alfabetización digital del alumnado es una necesidad. Para posibilitarla, se destacan entornos que fomenten el aprendizaje colaborativo y la autoría que se pueden favorecer y enriquecer usando las TIC. Nascimento y Knobel (2017, p. 83) mencionan seis estudios que abordaron las alfabetizaciones digitales "como una forma de brindar a los futuros maestros la oportunidad de aprender de manera colaborativa". En todos ellos, los alumnos experimentaban el medio digital desde la colaboración y reflexionaban sobre la alfabetización digital "desarrollando una comprensión profunda de las alfabetizaciones digitales que funcionará bien en los contextos del aula" (Nascimento y Knobel, 2017, p. 84). En línea con estos estudios, destacamos otro sobre cómo ir transformando la cultura de enseñanza y aprendizaje (en la universidad) fruto de implementar el proyecto Do It Yourself in Education: Expanding Digital Competence To Foster Student Agency And Collaborative Learning (Miño-Puigcercós et al., 2019).

- La dimensión prioritaria de la competencia digital metodológica es la comunicación y colaboración. Sobre sus beneficios, García-Valcárcel y Tejedor (2017, p. 141) destacan que "el desarrollo de habilidades de comunicación y de colaboración usando las TIC favorece el aprendizaje y el trabajo en grupo reflexionando, analizando y resolviendo problemas". Así, se impulsa el aprender de los otros y con los otros usando metodologías activas. En la práctica, esto requiere un replanteamiento especialmente del rol docente como uno de los desafíos más difíciles (Adams et al., 2017, p. 34). Por ejemplo, para favorecer este cambio, se subraya que "mediante la colaboración y el intercambio, los docentes usarán más la tecnología, mejorarán la CDD (...) y asumirán riesgos con sus nuevas habilidades" (Spiteri y Chang-Rundgren, 2017, p. 530).

- La ética y civismo digital es una dimensión emergente a contemplar durante la actividad educativa. Como prioritario se destaca el uso responsable, seguro y saludable de las tecnologías digitales. Después, la protección de los derechos a la intimidad personal y a la propia imagen. Y finalmente, la promoción del acceso a los recursos respetando la propiedad intelectual. Existen planteamientos en línea con esta temática. Gutiérrez y Tyner (2012, p. 38) resaltan la necesidad de "evitar centrarse en los conocimientos técnicos, y en los procedimientos de uso y manejo de dispositivos y programas, olvidando las actitudes y los valores". Ferrari (2012) enfatiza la actitud y el uso crítico de las tecnologías digitales en su definición de CDD. Y según Sancho-Gil et al. (2018, p. 43), "la formación no puede quedarse en la adquisición de habilidades tecnológicas aisladas, sino que es necesario generar una comprensión profunda y crítica de los entornos digitales".

- El uso de recursos digitales útiles para enseñar y aprender. Existen varios estudios sobre las potencialidades de estos recursos durante la práctica educativa. Camacho y Esteve (2017) y Herrington y Parker (2013, p. 608) señalan la importancia del uso de tecnologías emergentes como herramientas cognitivas para resolver 
problemas dentro de un entorno de aprendizaje auténtico. Romeu, Guitert, y Sangrà (2016) destacan el potencial de comunidades y redes como fuente de conocimiento. Y García-Valcárcel y Tejedor (2017, p. 153) inciden sobre el valor de las TIC en las estrategias de aprendizaje y su relación con el rendimiento durante la práctica educativa. En estos estudios, la tecnología es un instrumento al servicio de fines educativos (de Vries, 2016, citado en Castañeda et al., 2018, p. 14). Además, Selwyn (2013, p. 5) destaca que las tecnologías digitales adquieren un papel integral en el aprendizaje a lo largo de la vida.

- Finalmente, se apuntan tres acciones institucionales prioritarias a promover en los planes de estudio de los grados de Maestro. En la primera, se propone incorporar un módulo obligatorio de formación específica de CDD de como mínimo seis créditos. En la segunda, se plantea ir incorporando la CDD en los diferentes módulos de los planes de estudio, especialmente desde las asignaturas de didáctica. En la tercera, se sugiere ofertar una mención de CDD o, alternativamente, una o varias asignaturas optativas sobre la CDD. El traslado de estas acciones a la práctica educativa favorecería "prestar más atención al significado de la competencia digital profesional en la formación del profesorado" (Instefjord y Munthe, 2017, p. 44). Así, esta competencia se integraría mejor en los programas de formación del profesorado.

- En estos momentos, las universidades implicadas en el proyecto están rediseñando los contextos institucionales educativos. Por lo tanto, estamos pendientes de que todas estas percepciones puedan ser valoradas por sus comunidades universitarias y por la comunidad educativa en general. A su vez, esperamos que puedan concretarse en una propuesta para cada universidad y también en una propuesta interuniversitaria. Ahora bien, se requerirán otros proyectos para establecer procedimientos de desarrollo y evaluación. Todo ello, con la finalidad de poder asegurar que los estudiantes de los grados de Maestro alcancen la CDD al finalizar su formación de grado.

Una limitación de este estudio es que no pudimos incluir y examinar todos los aspectos posibles que influyen en el desarrollo de la CDD. Así, como el hecho de haber usado una muestra circunscrita en un territorio. Ahora bien, esperamos que estas aportaciones puedan tenerse en cuenta y aporten valor añadido al diseño de futuras propuestas formativas para los grados de Maestro. A título de cierre, una transformación de la universidad es inevitable destacando los desafíos en relación con repensar cómo este cambio afecta a la experiencia de aprendizaje de todos: de los estudiantes y del profesorado.

\section{Apoyos}

Agencia de Gestión de Ayudas Universitarias y de Investigación (AGAUR). Generalitat de Cataluña.

Grupo de investigación ESBRINA -Subjetividades, visualidades y entornos educativos contemporáneos- (2017 SGR 1248).

Red de excelencia REUNI+D - Red Universitaria de Investigación e Innovación Educativa. Cambios Sociales y Retos para la Educación en la Era Digital. EDU2015- 68718-REDT. 


\section{Referencias}

Adams, S., Cummins, M., Davis, A., Freeman, A., Hall, C., y Ananthanarayanan, V. (2017). NMC Horizon Report: 2017 Higher Education Edition. Austin, Texas: The New Media Consortium. Recuperado de https://goo.gl/8DsG2A

Batane, T., y Ngwako, A. (2017). Technology use by pre-service teachers during teaching practice: Are new teachers embracing technology right away in their first teaching experience? Australasian Journal of Educational Technology, 33(1), 58-61. doi: https:// doi.org/10.14742/ajet.2299

Camacho, M., y Esteve, F. (2017). El uso de las tabletas y su impacto en el aprendizaje. Una investigación nacional en centros de educación primaria. Revista de Educación, 379, 170-191. doi: https://doi.org/10.4438/1988-592X-RE-2017-379-366

Castañeda, L., Esteve, F., y Adell, J. (2018). ¿Por qué es necesario repensar la competencia docente para el mundo digital? RED. Revista de Educación a Distancia, 56, 2-20. doi: https://doi.org/10.6018/red/56/6

Cela-Ranilla, J. M., Esteve-González, V., Esteve-Mon, F., González-Martínez, J., y Gisbert-Cervera, M. (2017). El docente en la sociedad digital: una propuesta basada en la pedagogía transformativa y en la tecnología avanzada. Profesorado. Revista de Currículum y Formación de Profesorado, 21(1), 403-422.

Creswell, J. W. (2009). Mapping the field of mixed methods research. Journal of Mixed Methods Research, 3(2), 95-108. doi: https://doi.org/10.1177/1558689808330883

Departament d'Ensenyament de la Generalitat de Catalunya (DEGC) (2016). Resolución ENS/1356/2016, de 23 de mayo, por la que se da publicidad a la definición de la Competencia digital docente. DOGC, 7133. Recuperado de http://portaldogc.gencat. cat/utilsEADOP/PDF/7133/1500245.pdf

Departament d'Ensenyament de la Generalitat de Catalunya (DEGC) (2018). Competència digital docent del professorat de Catalunya. Barcelona: Servei de Comunicació i Publicacions. Recuperado de https://goo.gl/omDGjP

Domingo-Coscollola, M., Onsès-Segarra, J., y Sancho-Gil, J. M. (2018). La cultura DIY en educación primaria. Aprendizaje transdisciplinar, colaborativo y compartido en un Hub. Revista de investigación educativa, 36(2), 491-508.

Ferrari, A. (2012). Digital Competence in Practice: An Analysis of Frameworks. Luxembourg: Publications Office of the European Union. Recuperado de http://ftp.jrc.es/EURdoc/ JRC68116.pdf

García-Valcárcel, A., y Tejedor, F. J. (2017). Percepción de los estudiantes sobre el valor de las TIC en sus estrategias de aprendizaje y su relación con el rendimiento. Educación XX1, 20(2), 137-159. doi: https://doi.org/10.5944/educxx1.19035

Gudmundsdottir, G. B., y Hatlevik, O. E. (2018). Newly qualified teachers' professional digital competence: implications for teacher education. European Journal of Teacher Education, 41(2), 214-231. doi: https://doi.org/10.1080/02619768.2017.1416085

Gutiérrez, A., Palacios, A., y Torrego, L. (2010). Formar al profesorado inicialmente en habilidades y competencias en TIC: Perfiles de una experiencia colaborativa. Revista de Educación, 353, 267-293.

Gutiérrez, A., y Tyner, K. (2012). Media Education, Media Literacy and Digital Competence. Comunicar, 38(19), 31-39. doi: https://doi.org/10.3916/C38-2012-02-03 
Guzmán-Simón, F., García-Jiménez, E., y López-Cobo, I. (2017). Undergraduate Students' Perspectives on Digital Competence and Academic Literacy in a Spanish University. Computers in Human Behavior. 74, 196-204. doi: https://doi.org/10.1016/j. chb.2017.04.040

Hall, R., Atkins, L., y Fraser, J. (2014). Defining a Self-Evaluation Digital Literacy for Secondary Educators: the DigiLit Leicester Project. Research in Learning Technology, 22, 21440. doi: https://doi.org/10.3402/rlt.v22.21440

Herrada, R., y Herrada, G. (2011). Adaptación de los estudios de magisterio al EEES: Las TIC en los nuevos planes de estudio. Edutec-e. Revista electrónica de Tecnología Educativa, 36. doi: https://doi.org/10.21556/edutec.2011.36.405

Herrington, J., y Parker, J. (2013). Emerging technologies as cognitive tools for authentic learning. British Journal of Educational Technology, 44(4), 607-615. doi: https:/doi. org/10.1111/bjet.12048

Instefjord, E. J., y Munthe, E. (2017). Educating digitally competent teachers: A study of integration of professional digital competence in teacher education. Teaching and Teacher Education, 67, 37-45. doi: https://doi.org/10.1016/j.tate.2017.05.016

Instituto Nacional de Tecnologías Educativas y Formación del Profesorado (INTEF) (2017). Marco Común de Competencia Digital Docente Octubre 2017. Recuperado de https://bit.ly/2DUPvHa

International Society for Technology in Education (ISTE) (2018). ISTE Standards for Educators. Recuperado de https://www.iste.org/standards/for-educators

Kampylis, P., Punie, Y., y Devine, J. (2015). Promoting Effective Digital-Age Learning. A European Framework for Digitally-Competent Educational Organisations. EUR 27599 EN. doi: https://doi.org/10.2791/54070. Recuperado de https://goo.gl/o6CGsa

Krueger, R. A., y Casey, M. A. (2014). Focus groups: A practical guide for applied research. Thousand Oaks (CA): Sage Publications Ltd.

Krumsvik, R. J. (2014). Teacher educators' digital competence. Scandinavian Journal of Educational Research, 58(3), 269-280. doi: https://doi.org/10.1080/00313831.2012.726273

Losada, D., Valverde, J., y Correa, J. M. (2012). La tecnología educativa en la Universidad Pública Española. Píxel-Bit, Revista de Medios y Educación, 41, 133-148.

Miño-Puigcercós, R., Domingo-Coscollola, M., y Sancho-Gil, J. M. (2019). Transforming the Teaching and Learning Culture in Higher Education from a DIY perspective. Educación XX1, 22(1), 139-160. doi: https://doi.org/10.5944/educXX1.20057

Nascimento, A. K. O., y Knobel, M. (2017). What's to be learned? Nordic Journal of Digital Literacy, 12(3), 67-88. doi: https://doi.org/10.18261/issn.1891-943x-2017-03-03

Paredes, J., Guitert M., y Rubia B. (2015). La innovación y la tecnología educativa como base de la formación inicial del profesorado para la renovación de la enseñanza. Revista Latinoamericana de Tecnología Educativa, 14(1), 101-114.

Redecker, C., y Punie, Y. (2017). European Framework for the Digital Competence of Educators: DigCompEdu. Luxembourg: Publications Office of the European Union. Recuperado de https://goo.gl/YovFy4

Romeu, T., Guitert, M., y Sangrà, A. (2016). Teacher collaboration network in Higher Education: reflective visions from praxis. Innovations in Education and Teaching International, 53(6), 592-604. doi: https://doi.org/10.1080/14703297.2015.1025807 
Ruiz, I., Rubia, B., Anguita, R., y Fernández, E. (2010). La formación de los futuros maestros y la integración de las TIC en la educación: anatomía de un desencuentro. Revista de Educación, 352, 149-178.

Sancho-Gil, J. M., Bosco, A., Alonso, C., y Sánchez, J. A. (2015). Formación del profesorado en Tecnología Educativa: de cómo las realidades generan los mitos. Revista Latinoamericana de Tecnología Educativa, 14(1), 17-29.

Sancho-Gil, J. M., Sánchez-Valero, J. A., y Domingo-Coscollola, M. (2017). Research-based insights on initial teacher education in Spain. European Journal of Teacher Education, 40(3), 310-325. doi: https://doi.org/10.1080/02619768.2017.1320388

Sancho-Gil, J. M., Ornellas, A., y Arrazola, J. (2018). La situación cambiante de la universidad en la era digital. RIED. Revista Iberoamericana de Educación a Distancia, 21(2), 31-49. doi: http://dx.doi.org/10.5944/ried.21.2.20673

Selwyn, N. (2013). Education in a Digital World: Global Perspectives on Technology and Education. Londres: Routledge.

Spiteri, M., y Chang-Rundgren, S. N. (2017). Maltese primary teachers' digital competence: implications for continuing professional development. European Journal of Teacher Education, 40(4), 521-534. doi: https://doi.org/10.1080/02619768.2017.1342242

Suárez-Rodríguez, J. M., Almerich, G., Gargallo-López, B., y Aliaga, F. M. (2013). Las competencias del profesorado en TIC: estructura básica, Educación XX1, 16(1), 39-62. doi: https://doi.org/10.5944/educxx1.16.1.716

Tondeur, J., Scherer, R., Siddiq, F., y Baran, E. (2017). A comprehensive investigation of TPACK within pre-service teachers' ICT profiles: Mind the gap! Australasian Journal of Educational Technology, 33(3), 46-60. doi: https://doi.org/10.14742/ajet.3504

Tourón, J., Martín, D., Navarro, E., Pradas, S., e Íñigo, V. (2018). Validación de constructo de un instrumento para medir la competencia digital docente de los profesores (CDD). Revista Española de Pedagogía, 76(269), 25-54. doi: https://doi.org/10.22550/ REP76-1-2018-02

UNESCO (2011). UNESCO ICT Competency Framework for Teachers. París: UNESCO. Recuperado de http://unesdoc.unesco.org/images/0021/002134/213475e.pdf

Fecha de recepción: 24 de mayo de 2018.

Fecha de revisión: 5 de junio de 2018.

Fecha de aceptación: 4 de julio de 2019. 